\title{
ON DOUBLE COSINE, SINE, AND WALSH SERIES WITH MONOTONE COEFFICIENTS
}

\author{
FERENC MÓRICZ
}

(Communicated by J. Marshall Ash)

\begin{abstract}
We extend from one-dimensional to two-dimensional series the results by Hardy and Littlewood [6] on the $L^{r}$-integrability of the sum $f$ and the results by Stechkin [10] on the $L^{1}$-integrability of the maximum partial sum $M^{*}$ in the case of cosine and sine series with monotone coefficients. Among others, we prove that the $L^{r}$-integrability of $f$ and $M^{*}$ is essentially equivalent for $r>1$ in the two-dimensional setting, too. Simultaneously, we extend our earlier results in [7] from one-dimensional to two-dimensional Walsh series.
\end{abstract}

\section{INTRODUCTION}

We will consider the double cosine series

$$
\sum_{j=0}^{\infty} \sum_{k=0}^{\infty} \lambda_{j} \lambda_{k} a_{j k} \cos j x \cos k y
$$

and the double sine series

$$
\sum_{j=1}^{\infty} \sum_{k=1}^{\infty} a_{j k} \sin j x \sin k y
$$

on the positive quadrant $[0, \pi] \times[0, \pi]$ of the two-dimensional torus, where $\lambda_{0}=\frac{1}{2}$ and $\lambda_{j}=1$ for $j \geq 1$, and the double Walsh series

$$
\sum_{j=0}^{\infty} \sum_{k=0}^{\infty} a_{j k} w_{j}(x) w_{k}(y)
$$

on the unit square $[0,1) \times[0,1)$. As to the definition of the Walsh functions $w_{j}(x)$ in the Paley enumeration, we refer the reader to [1, p. 60]. Usually we assume that the real coefficients $a_{j k}$ are such that

$$
a_{j k} \geq 0, \quad \Delta_{10} a_{j k} \geq 0, \quad \Delta_{01} a_{j k} \geq 0, \quad \Delta_{11} a_{j k} \geq 0
$$

Received by the editors May 16, 1989 and, in revised form, August 2, 1989.

1980 Mathematics Subject Classification (1985 Revision). Primary 42A32; Secondary 42B05.

Key words and phrases. Rectangular partial sum, maximum partial sum, pointwise convergence and convergence in $L^{r}$-norm in Pringsheim's sense, Hardy's inequalities for double integrals and double sequences. 
where

$$
\begin{aligned}
& \Delta_{10} a_{j k}=a_{j k}-a_{j+1, k}, \\
& \Delta_{01} a_{j k}=a_{j k}-a_{j, k+1}, \\
& \Delta_{11} a_{j k}=a_{j k}-a_{j+1, k}-a_{j, k+1}+a_{j+1, k+1} \quad(j, k \geq 0) .
\end{aligned}
$$

The second and third inequalities in (1.4) express the fact that $\left\{a_{j k}\right\}$ as a sequence is monotone decreasing in both $j$ and $k$. If $\left\{a_{j k}\right\}$ is a null sequence in the sense that

$$
a_{j k} \rightarrow 0 \text { as } \max (j, k) \rightarrow \infty,
$$

then the first three inequalities in (1.4) follow from the fourth one.

We denote by $s_{m n}(x, y)$ the rectangular partial sums of series (1.1)-(1.3). For example, in the case of series (1.1),

$$
s_{m n}(x, y)=\sum_{j=0}^{m} \sum_{k=0}^{n} \lambda_{j} \lambda_{k} a_{j k} \cos j x \cos k y \quad(m, n \geq 0) .
$$

Applying double summation by parts, it is routine to show that if conditions (1.4) and (1.5) are satisfied, then series (1.1)-(1.3) converge in Pringsheim's sense, i.e., the finite limit

$$
s_{m n}(x, y) \rightarrow f(x, y) \text { as } \min (m, n) \rightarrow \infty
$$

exists, except possibly at $x=0$ or $y=0$ in the case of series (1.1) and (1.3). We set

$$
M^{*}(x, y)=\sup _{m, n}\left|s_{m n}(x, y)\right|
$$

for the maximum partial sum of series (1.1)-(1.3) and

$$
S_{r}=\left\{\sum_{j} \sum_{k}\left|a_{j k}\right|^{r}(j+1)^{r-2}(k+1)^{r-2}\right\}^{1 / r} \quad(r>0)
$$

where the summations are extended over $j, k \geq 0$ in the case of series (1.1) and (1.3), and over $j, k \geq 1$ in the case of series (1.2). By Hölder's inequality,

$$
S_{r} \leq\left\{\sum_{j=1}^{\infty} \frac{1}{j^{2}}\right\}^{2\left(1 / r-1 / r^{\prime}\right)} S_{r^{\prime}} \quad\left(0<r<r^{\prime}<\infty\right) .
$$

In the sequel, by $\|g\|_{r}$ we mean the $L^{r}$-norm $\left\{\int_{0}^{\pi} \int_{0}^{\pi}|g(x, y)|^{r} d x d y\right\}^{1 / r}$ in the case of series (1.1) and (1.2), and $\left\{\int_{0}^{1} \int_{0}^{1}|g(x, y)|^{r} d x d y\right\}^{1 / r}$ in the case of series (1.3). Furthermore, by $C$ we denote a positive absolute constant which may be different from place to place; by $C_{r}, \widetilde{C}_{r}$ we denote positive constants depending only on $r(\geq 1)$. 


\section{AN UPPER ESTIMATE IN CASE $r \geq 1$}

The following results are extensions of those by Stechkin [10] (in the case of $r=1$ ) and the present author [7] from one-dimensional to two-dimensional series.

Theorem 1. If condition (1.4) is satisfied and $r \geq 1$, then

$$
\left\|M^{*}\right\|_{r} \leq C_{r} S_{r} .
$$

This implies immediately the following

Corollary 1. If condition (1.4) is satisfied and $S_{r}<\infty$ for some $r \geq 1$, then the sum $f$ is $L^{r}$-integrable and

$$
\left\|s_{m n}-f\right\|_{r} \rightarrow 0 \text { as } \min (m, n) \rightarrow \infty .
$$

In particular, series (1.1)-(1.3) are Fourier series of their sums.

We need the two-parameter version of an inequality of Hardy and Littlewood [5].

Lemma 1. If $a_{j k} \geq 0, r \geq 1, c_{1}>1$, and $c_{2}>1$, then

$$
\sum_{p=1}^{\infty} \sum_{q=1}^{\infty} \frac{1}{p^{c_{1}} q^{c_{2}}}\left(\sum_{j=1}^{p} \sum_{k=1}^{q} a_{j k}\right)^{r} \leq\left(\frac{r^{2}}{\left(c_{1}-1\right)\left(c_{2}-1\right)}\right)^{r} \sum_{j=1}^{\infty} \sum_{k=1}^{\infty} a_{j k}^{r} j^{r-c_{1}} k^{r-c_{2}} .
$$

Lemma 1 is a discrete version of Lemma 2 below (cf. [11, vol. 2, p. 129]).

Proof of Theorem 1. We prove it in a more general setting. Let $\left\{f_{j}(x): j=\right.$ $1,2, \ldots\}$ be a sequence of real-valued functions defined on the interval $[0,1]$. Let

$$
s_{m n}(x, y)=\sum_{j=1}^{m} \sum_{k=1}^{n} a_{j k} f_{j}(x) f_{k}(y) \quad(m, n \geq 1)
$$

where the $a_{j k}$ satisfy condition (1.4) and let $M^{*}$ be defined by (1.6). We assume that the $f_{j}(x)$ are uniformly bounded:

$$
\left|f_{j}(x)\right| \leq B(<\infty) \quad(j \geq 1 ; 0 \leq x \leq 1)
$$

and that the sum of the first $m$ functions $f_{j}(x)$ (the so-called Dirichlet kernel in the case of series (1.1)) is such that

$$
\left|D_{m}(x)\right|=\left|\sum_{j=1}^{m} f_{j}(x)\right| \leq \frac{C}{x} \quad(m \geq 1 ; 0<x \leq 1) .
$$

As is well known, inequality (2.3) is satisfied in the case of cosine and sine functions (see, e.g., [11, vol. 1, p. 51]) and Walsh functions [3].

Given $0<x, y<1$, define the positive integers $p$ and $q$ such that

$$
\frac{1}{p+1}<x \leq \frac{1}{p} \text { and } \frac{1}{q+1}<y \leq \frac{1}{q} \text {. }
$$


Using (2.2) we can estimate in a trivial manner

$$
\begin{aligned}
\left|s_{m n}(x, y)\right| \leq & B^{2} \sum_{j=1}^{p-1} \sum_{k=1}^{q-1} a_{j k}+B \sum_{k=1}^{q-1}\left|\sum_{j=p}^{m} a_{j k} f_{j}(x)\right| \\
& +B \sum_{j=1}^{p-1}\left|\sum_{k=q}^{n} a_{j k} f_{k}(y)\right|+\left|\sum_{j=p}^{m} \sum_{k=q}^{n} a_{j k} f_{j}(x) f_{k}(y)\right|
\end{aligned}
$$

It may happen that some of these sums are empty. As usual, an empty sum means zero.

A summation by parts gives

$$
\sum_{j=p}^{m} a_{j k} f_{j}(x)=-a_{p k} D_{p-1}(x)+\sum_{j=p}^{m-1} D_{j}(x) \Delta_{10} a_{j k}+a_{m k} D_{m}(x)
$$

whence, by (1.4) and (2.3),

$$
\left|\sum_{j=p}^{m} a_{j k} f_{j}(x)\right| \leq \frac{2 C}{x} a_{p k} .
$$

By (1.4) and (2.4),

$$
\sum_{k=1}^{q-1}\left|\sum_{j=p}^{m} a_{j k} f_{j}(x)\right| \leq 4 C p \sum_{k=1}^{q-1} a_{p k} \leq 4 C \sum_{j=1}^{p} \sum_{k=1}^{q-1} a_{j k} .
$$

Analogously, we have

$$
\sum_{j=1}^{p-1}\left|\sum_{k=q}^{n} a_{j k} f_{k}(y)\right| \leq 4 C \sum_{j=1}^{p-1} \sum_{k=1}^{q} a_{j k} .
$$

Finally, a double summation by parts yields

$$
\begin{aligned}
\sum_{j=p}^{m} \sum_{k=q}^{n} a_{j k} f_{j}(x) f_{k}(y)= & \sum_{j=p}^{m-1} \sum_{k=q}^{n-1} D_{j}(x) D_{k}(y) \Delta_{11} a_{j k} \\
& +\sum_{j=p}^{m-1} D_{j}(x) D_{n}(y) \Delta_{10} a_{j n}-\sum_{j=p}^{m-1} D_{j}(x) D_{q}(y) \Delta_{10} a_{j q} \\
& +\sum_{k=q}^{n-1} D_{m}(x) D_{k}(y) \Delta_{01} a_{m k}-\sum_{k=q}^{n-1} D_{p}(x) D_{k}(y) \Delta_{01} a_{p k} \\
& +a_{m n} D_{m}(x) D_{n}(y)-a_{p n} D_{p}(x) D_{n}(y)-a_{m q} D_{m}(x) D_{q}(y) \\
& +a_{p q} D_{p}(x) D_{q}(y) .
\end{aligned}
$$


Hence, by means of (1.4), (2.3), and (2.4),

$$
\begin{aligned}
\left|\sum_{j=p}^{m} \sum_{k=q}^{n} a_{j k} f_{j}(x) f_{k}(y)\right| & \leq \frac{4 C^{2}}{x y} a_{p q} \\
& \leq 16 C^{2} p q a_{p q} \leq 16 C^{2} \sum_{j=1}^{p} \sum_{k=1}^{q} a_{j k} .
\end{aligned}
$$

Combining (2.5)-(2.8) furnishes

$$
\int_{0}^{1} \int_{0}^{1}\left[M^{*}(x, y)\right]^{r} d x d y \leq C_{r} \sum_{p=1}^{\infty} \sum_{q=1}^{\infty} \frac{1}{p(p+1) q(q+1)}\left(\sum_{j=1}^{p} \sum_{k=1}^{q} a_{j k}\right)^{r} .
$$

By virtue of Lemma 1, this implies that (2.1) is proved.

\section{A LOWER ESTimate IN CASE $r>1$}

In case (i) of Theorem 2 below we assume $a_{00}=a_{j 0}=a_{0 k}=0 \quad(j, k \geq 1)$, and accordingly we require the fulfillment of (1.4) only for $j, k \geq 1$ this time.

Theorem 2. If conditions (1.4) and (1.5) are satisfied and the sums $f$ of series (1.1)-(1.3) belong to $L^{r}$ for some $r>1$, then

(i) in the case of series (1.1) and (1.2),

$$
\left(\sum_{j=2}^{\infty} \sum_{k=2}^{\infty} a_{j k}^{r}(j k)^{r-2}\right)^{1 / r} \leq C_{r}\|f\|_{r}
$$

(ii) in the case of series (1.3),

$$
\left(\sum_{j=1}^{\infty} \sum_{k=1}^{\infty} a_{j k}^{r}(j k)^{r-2}\right)^{1 / r} \leq C_{r}\|f\|_{r}
$$

These results are extensions of those by Hardy and Littlewood [6] (see also [11, vol. 2, p. 129]) and by the present author [7] from one-dimensional to twodimensional series. We note that case (i) in Theorem 2 is a direct consequence of the results of [2] by D'yachenko and [8] by the present author.

Due to the first two conditions in (1.4) and condition (1.5), the first row in series (1.1) corresponding to $k=0$ (while dropping the factor $\lambda_{0}=\frac{1}{2}$ ) converges, except possibly at $x=0$ :

$$
\sum_{j=0}^{\infty} \lambda_{j} a_{j 0} \cos j x=f_{1}(x), \text { say. }
$$

By virtue of the one-dimensional result in [6], for $r>1$ we have

$$
\widetilde{C}_{r} \sum_{j=2}^{\infty} a_{j 0}^{r} j^{r-2} \leq \int_{0}^{\pi}\left|f_{1}(x)\right|^{r} d x \leq C_{r} \sum_{j=0}^{\infty} a_{j 0}^{r}(j+1)^{r-2}
$$


Likewise, the first column in series (1.1) corresponding to $j=0$ converges, except possibly at $y=0$ :

$$
\sum_{k=0}^{\infty} \lambda_{k} a_{0 k} \cos k y=f_{2}(y), \text { say, }
$$

and we have an analogous pair of inequalities as above.

Similar estimates hold in the case of series (1.2) and (1.3) (see [7] concerning $(1.3)$ ).

We agree to denote by $f_{1}(x)$ and $f_{2}(y)$ the sum of the first row and the first column, respectively, in each case of series (1.1) (dropping $\left.\lambda_{0}=1 / 2\right),(1.2)$ (dropping $\sin y$ or $\sin x$, respectively), and (1.3). Combining Theorems 1 and 2 with the corresponding one-dimensional results yields the following

Corollary 2. Assume that conditions (1.4) and (1.5) are satisfied and $r>1$. Then the sums $f(x, y), f_{1}(x)$, and $f_{2}(y)$ belong to $L^{r}$ if and only if $S_{r}<\infty$.

In particular, $M^{*}$ belongs to $L^{r}$ if and only if $f, f_{1}, f_{2}$ belong to $L^{r}$.

We need the two-dimensional version of an inequality of Hardy [4] (see also [11, Vol. 1, p. 20]).

Lemma 2. Let $r \geq 1, c_{1}<r-1, c_{2}<r-1, f(x, y)$ be a nonnegative function defined for $x, y>0$ and

$$
F(x, y)=\int_{0}^{x} \int_{0}^{y} f(u, v) d u d v \quad(x, y>0) .
$$

If $f^{r}(x, y) x^{c_{1}} y^{c_{2}}$ is integrable over $(0, \infty) \times(0, \infty)$, then so is $\{F(x, y) / x y\}^{r}$ $\times x^{c_{1}} y^{c_{2}}$ and

$$
\begin{aligned}
I & =\left\{\int_{0}^{\infty} \int_{0}^{\infty}\left\{\frac{F(x, y)}{x y}\right\}^{r} x^{c_{1}} y^{c_{2}} d x d y\right\}^{1 / r} \\
& \leq \frac{r^{2}}{\left(r-c_{1}-1\right)\left(r-c_{2}-1\right)}\left\{\int_{0}^{\infty} \int_{0}^{\infty} f^{r}(x, y) x^{c_{1}} y^{c_{2}} d x d y\right\}^{1 / r}
\end{aligned}
$$

Proof of Lemma 2. The next elegant proof is due to Professor Alberto Torchinsky, who was so kind to allow the author to publish it here.

Making use of the substitutions $u=x u^{\prime}$ and $v=y v^{\prime}$ (omitting primes in the resulting integral) and then applying the integral version of Minkowski's inequality yields

$$
\begin{aligned}
I & =\left\{\int_{0}^{\infty} \int_{0}^{\infty}\left\{\int_{0}^{1} \int_{0}^{1} f(x u, y v) d u d v\right\}^{r} x^{c_{1}} y^{c_{2}} d x d y\right\}^{1 / r} \\
& \leq \int_{0}^{1} \int_{0}^{1}\left\{\int_{0}^{\infty} \int_{0}^{\infty} f^{r}(x u, y v) x^{c_{1}} y^{c_{2}} d x d y\right\}^{1 / r} d u d v .
\end{aligned}
$$

Performing the substitutions $x u=x^{\prime}$ and $y v=y^{\prime}$ (omitting primes again) results in

$$
I \leq \int_{0}^{1} \int_{0}^{1} \frac{d u d v}{u^{\left(c_{1}+1\right) / r} v^{\left(c_{2}+1\right) / r}}\left\{\int_{0}^{\infty} \int_{0}^{\infty} f^{r}(x, y) x^{c_{1}} y^{c_{2}} d x d y\right\}^{1 / r} .
$$

Hence (3.4) follows immediately. 
Proof of Theorem 2. (i) We proved in [8] that, under conditions (1.4) and (1.5), if $f \in L$ then the double cosine or sine series defining $f$ is the Fourier series of $f$. Then we apply [2, Theorem 1] in order to obtain (3.1).

(ii) Let $p$ and $q$ be positive integers, and let $F(x, y)$ be defined by (3.3). It is known (see [9]) that if $f \in L$, in particular if $f \in L^{r}$, then series (1.3) is the Walsh-Fourier series of $f$. By the first three conditions in (1.4), we conclude that

$$
\begin{aligned}
F\left(2^{-p}, 2^{-q}\right) & =2^{-p-q} \int_{0}^{1} \int_{0}^{1} f(x, y) D_{2^{p}-1}(x) D_{2^{q}-1}(y) d x d y \\
& =2^{-p-q} \sum_{j=0}^{2^{p}-1} \sum_{k=0}^{2^{q}-1} a_{j k} \geq a_{2^{p}-1,2^{q}-1} .
\end{aligned}
$$

Here we also used the fact [3] that

$$
D_{2^{p}-1}(x)= \begin{cases}2^{p} & \text { for } 0 \leq x<2^{-p} \\ 0 & \text { for } 2^{-p} \leq x<1\end{cases}
$$

On the one hand, by (3.4) and (3.5),

$$
\begin{aligned}
& \sum_{p=1}^{\infty} \sum_{q=1}^{\infty} a_{2^{p}-1,2^{q}-1}^{r} 2^{(p+q)(r-1)} \\
& \quad \leq \sum_{p=1}^{\infty} \sum_{q=1}^{\infty} \int_{2^{-p}}^{2^{-p+1}} \int_{2^{-q}}^{2^{-q+1}}\left\{\frac{G(x, y)}{x y}\right\}^{r} d x d y \leq\left(\frac{r}{r-1}\right)^{2 r}\|f\|_{r}^{r},
\end{aligned}
$$

where

$$
G(x, y)=\int_{0}^{x} \int_{0}^{y}|f(u, v)| d u d v \quad(x, y>0) .
$$

On the other hand, by monotonicity,

$$
\begin{aligned}
\sum_{j=1}^{\infty} & \sum_{k=1}^{\infty} a_{j k}^{r}(j k)^{r-2} \\
& \leq \sum_{p=1}^{\infty} \sum_{q=1}^{\infty} a_{2^{p}-1,2^{q}-1}^{r} \sum_{j=2^{p}-1}^{2^{p+1}-2} \sum_{k=2^{q}-1}^{2^{q+1}-2}(j k)^{r-2} \\
& \leq 4^{|r-2|} \sum_{p=1}^{\infty} \sum_{q=1}^{\infty} a_{2^{p}-1,2^{q}-1}^{r} 2^{(p+q)(r-1)} .
\end{aligned}
$$

Combining (3.6) and (3.7) yields that (3.2) is proved.

\section{A LOWER ESTIMATE IN CASE $r=1$}

Theorem 2 is no longer true if $r=1$. However, if we define

$$
M(x, y)=\sup _{p, q \geq 0}\left\{s_{00}(x, y), s_{2^{p}, 0}(x, y), s_{0,2^{q}}(x, y), s_{2^{p}, 2^{q}}(x, y)\right\}
$$


in the case of series (1.1),

$$
M(x, y)=\sup _{p, q \geq 0} s_{2^{p}, 2^{q}}(x, y)
$$

in the case of series (1.2), and

$$
M(x, y)=\sup _{p, q \geq 0} s_{2^{p}-1,2^{q}-1}(x, y)
$$

in the case of series (1.3), and substitute $M$ for $f$ in (3.1) and (3.2), then these inequalities hold for $r=1$.

Theorem 3. If $a_{j k} \geq 0$, then $\|M\|_{1} \geq C S_{1}$.

Putting Theorems 1 and 3 together yields the following

Corollary 3. Assume condition (1.4) is satisfied. Then $M^{*}$ and $M$ belong to $L^{1}$ if and only if $S_{1}<\infty$.

In particular, the $L^{1}$-integrability of $M^{*}$ and $M$ is equivalent.

Proof of Theorem 3. We prove it in the case of series (1.3). Clearly, if $0 \leq x<$ $2^{-p}$ and $0 \leq y<2^{-q}$ then by definition of the Walsh functions

$$
s_{2^{p}-1,2^{q}-1}(x, y)=\sum_{j=0}^{2^{p}-1} \sum_{k=0}^{2^{q}-1} a_{j k} \quad(p, q \geq 0) .
$$

Hence

$$
\begin{aligned}
\|M\|_{1} & \geq \sum_{p=0}^{\infty} \sum_{q=0}^{\infty} 2^{-p-q-2} \sum_{j=0}^{2^{p}-1} \sum_{k=0}^{2^{q}-1} a_{j k} \\
& =\sum_{j=0}^{\infty} \sum_{k=0}^{\infty} a_{j k} \sum_{p: 2^{p} \geq j+1} 2^{-p-1} \sum_{q: 2^{q} \geq k+1} 2^{-q-1} \geq C S_{1} .
\end{aligned}
$$

Analogous straightforward estimates show that (4.1) holds in the case of series (1.1) and (1.2), too.

\section{ACKNOWLEDGMENT}

The author expresses his gratitude to Professor Alberto Torchinsky for valuable discussions over this topic and to the referee for calling his attention to paper [2].

\section{REFERENCES}

1. G. Alexits, Convergence problems of orthogonal series, Pergamon Press, New York-OxfordParis, 1961.

2. M. I. D'yachenko, On the convergence of double trigonometric series and Fourier series with monotone coefficients, Math. USSR-Sb. 57 (1987), pp. 57-75.

3. N. J. Fine, On the Walsh functions, Trans. Amer. Math. Soc. 65 (1949), pp. 372-414.

4. G. H. Hardy, Notes on some points in the integral calculus (LXIV), Messenger for Math. 57 (1928), pp. 12-16. 
5. G. H. Hardy and J. E. Littlewood, Elementary theorems concerning power series with positive coefficients and moment constants of positive functions, J. Reine Angew. Math. 157 (1927), pp. 141-158.

6. Some new properties of Fourier constants, J. London Math. Soc. 6 (1931), pp. 3-9.

7. F. Móricz, On Walsh series with coefficients tending monotonically to zero, Acta. Math. Acad. Sci. Hungar. 38 (1981), pp. 183-189.

8. __, On the integrability of double cosine and sine series. I, J. Math. Anal. Appl. (submitted).

9. F. Móricz, F. Schipp, and W. Wade, On the integrability of double Walsh series with special coefficients, Michigan Math. J. (submitted).

10. S. B. Stechkin, On power series and trigonometric series with monotone coefficients (in Russian), Uspekhi Mat. Nauk 18(1) (1963), pp. 173-180.

11. A. Zygmund, Trigonometric series, Cambridge Univ. Press, Cambridge, 1959.

Bolyai Institute, University of Szeged, ARadi Vértanúk tere 1, 6720 Szeged, Hungary 